\title{
A CLASS OF INFINITE SOLUBLE GROUPS WITH AN A-GROUP CONDITION
}

\author{
by M. J. TOMKINSON
}

(Received 10 January, 1979)

Finite soluble groups in which all the Sylow subgroups are abelian were first investigated by Taunt [8] who referred to them as $A$-groups. Locally finite groups with the same property have been considered by Graddon [2]. By the use of Sylow theorems it is clear that every section (homomorphic image of a subgroup) of an $\boldsymbol{A}$-group is also an A-group and hence every nilpotent section of an A-group is abelian. This is the characterization that we use here in considering groups which are not, in general, periodic.

We consider the class $\mathfrak{S}_{1}$ of soluble groups having a finite (normal) series in which the factors are abelian groups of finite rank whose torsion subgroup satisfies the minimal condition [5, p. 137]. We define an $\mathfrak{S}_{1}-A$-group to be an $\widetilde{S}_{1}$-group in which every nilpotent section is abelian. We shall see that this condition is very restrictive. It follows immediately that every locally nilpotent and every residually nilpotent section is abelian. Every polycyclic group has a subgroup of finite index which is residually a finite $p$-group [6] and so a polycyclic $A$-group is abelian-by-finite. This result also holds for $\mathfrak{S}_{1}$-groups.

THEOREM 1. An $\mathfrak{S}_{1}-A$-group is abelian-by-finite.

Proof. The $\widetilde{S}_{1}-A$-group $G$ has a normal series

$$
1=A_{0}<A_{1}<\ldots<A_{k}=G
$$

in which the factors are

(I) an abelian p-group with the minimal condition, or

(II) a torsion-free abelian group which is rationally irreducible (as a $G$-module).

By induction, we may assume that $G$ has a normal abelian subgroup $A$ satisfying (I) or (II) and $G / A$ is abelian-by-finite. Let $H / A$ be an abelian normal subgroup with finite index in $G / A$.

Case I. Let $A_{n}=\Omega_{n}(A)$ and $C=C_{H}\left(A_{1}\right)$. Then $G / C$ is finite and $C$ centralizes $A_{n+1} / A_{n}$ for each $n$. Thus $C$ is hypercentral and hence abelian and $G$ is abelian-by-finite.

Case II. Let $C=C_{G}(A)$; then $\bar{G}=G / C$ is a soluble irreducible $\mathbf{Q}$-linear group and so has a free abelian normal subgroup $F / C$ of finite index [4, Corollary 1 to Lemma 5.29.1]. Since $G \in \mathbb{S}_{1}, F / C$ has finite rank and, in particular, $\bar{G}$ is polycyclic.

Let $a \in A$ and $M=\left\langle a^{G}\right\rangle$ so that $M$ is a finitely generated $\mathbf{Z} G$-module. Thus $M$ contains a free abelian subgroup $X$ such that $M / X$ is a $\Pi$-group for some finite set $\Pi$ of primes [5, Corollary 1 to Lemma 9.53]. If $p \notin \Pi$, then $M^{p^{n}} \cap X=X^{p^{n}}$ and so $\bigcap_{n=1}^{\infty} M^{p^{n}} \cap X=1$. Hence $\bigcap_{n=1}^{\infty} M^{p^{n}}=1$.

Let $K=C_{G}\left(M / M^{p}\right)$; then $K$ centralizes $M^{p^{n}} / M^{p^{n+1}}$, for each $n$. If $x \in K$, then $M\langle x\rangle$ is residually nilpotent and hence abelian. Thus $x$ centralizes $M$ and so $K=C_{G}(M)$. But then 
$M \leqslant C_{\mathrm{A}}(K)$ and, since $A$ is rationally irreducible, we have $C_{\mathrm{A}}(K)=A$ and so $A \leqslant \zeta(K)$. So $H \cap K$ is nilpotent and hence abelian and, since $G / H \cap K$ is finite, the proof is complete.

Theorem 2. If $G$ is an $\mathcal{S}_{1}-A$-group, then $G / Z$ satisfies the minimal condition.

Proof. Let $A$ be an abelian normal subgroup of $G$ containing $Z$ such that $G / A$ is finite. It is clearly sufficient to show that $A / Z$ satisfies the minimal condition. We show that $A / Z$ is periodic and that $\varpi(A / Z)$ is finite.

Let $a \in A$; then $X=\left\langle a^{G}\right\rangle$ is a finitely generated abelian normal subgroup of $G$ and so there is an integer $r$ such that $X^{r}$ is a free abelian normal subgroup of $G$. Let $G / A=\left\langle t_{1}, \ldots, t_{k}\right\rangle$ with $t_{i}=t_{i} A$ a $p_{i}$-element. Then $X^{r}\left\langle t_{i}\right\rangle /\left(X^{r}\right)^{p_{i}{ }^{n}}$ is nilpotent. Thus $X^{r}\left\langle t_{i}\right\rangle$ is residually nilpotent and hence abelian. Thus $C_{G}\left(X^{r}\right) \geqslant A\left\langle t_{1}, \ldots, t_{k}\right\rangle=G$ and so $X^{r} \leqslant Z$. In particular $a^{r} \in Z$ and so $A / Z$ is periodic.

Let $p \in \varpi(A / Z)$ and let $\bar{a}=a Z$ be an element of $A / Z$ of order $p$. There is an element $x \in G$ such that $[a, x] \neq 1$. Also $[a, x]^{p}=\left[a^{p}, x\right]=1$ and so $p \in \varpi(G)$. Since $\varpi(G)$ is finite, $\varpi(A / Z)$ is finite, as required.

Corollary 3. A polycyclic A-group is centre-by-finite.

It should be noted that $\widetilde{S}_{1}-A$-groups need not be centre-by-finite as the group obtained by taking the split extension of $C_{3^{\infty}}$ by its involution automorphism is an A-group with trivial centre.

Also, neither of Theorems 1 and 2 hold for $\widetilde{S}_{0}$-groups in which all nilpotent sections are abelian. For, let $p_{1}, p_{2}, \ldots$ and $q_{1}, q_{2}, \ldots$ be two sequences of distinct primes such that $q_{i} \mid p_{i}-1$. If $G_{i}$ is a nonabelian group of order $p_{i} q_{i}$ and $G=\operatorname{Dr}_{i=1}^{\infty} G_{i}$, then $G$ is an $\Im_{0}$-group in which all the nilpotent sections are abelian. $G$ has trivial centre and is not abelian-byfinite.

Theorem 4. If $G$ is an $\mathfrak{S}_{1}-A$-group, then $G^{\prime} \cap Z=1$.

Proof. Let $x \in G^{\prime} \cap Z$; then there is a finitely generated subgroup $H$ of $G$ such thal $x \in H^{\prime} \cap \zeta(H)$. Since $G$ is abelian-by-finite (Theorem 1$), H$ is polycyclic and so is residually finite. But by the result for finite soluble groups [8], $H^{\prime} \cap \zeta(H)$ is contained ir every normal subgroup of finite index in $H$ and therefore $H^{\prime} \cap \zeta(H)=1$. Hence $x=1$ anc so $G^{\prime} \cap Z=1$.

Combining this result with Theorem 2, we have

Corollary 5. If $G$ is an $\widetilde{S}_{1}-A$-group, then $G^{\prime}$ satisfies the minimal condition.

Corollary 6. If $G$ is an $\widetilde{S}_{1}-A$-group and $G^{(k)}$ is any term of the derived series of $G$ then $G / G^{(k)}$ is also an $\mathfrak{S}_{1}$-group.

The significance of Corollary 6 is that it enables us to consider certain factor group: of $G$ and use induction arguments. In general, if $G$ is an $\widetilde{S}_{1}$-group, $G / G^{\prime}$ need not be at $\widetilde{S}_{1}$-group. For, let $A=\mathbf{Z} \oplus \mathbf{Q}$ and extend $A$ by its automorphism $\alpha$ which maps $(n, q)$ tc $(n, q+n)$. Then $G$ is a torsion-free $\widetilde{S}_{1}$-group but $G^{\prime}=\{(0, n): n \in \mathbf{Z}\} \leqslant A$, so that $G / G$ contains a subgroup isomorphic to $\mathbf{Q} / \mathbf{Z}$, which is not an $\widetilde{S}_{1}$-group. 
We now turn our attention to complementation theorems involving A-groups. If $\mathfrak{\Im}$ is a saturated formation of finite soluble groups and $G$ is a finite soluble group with abelian §-residual $G^{\Re}$, then $G$ splits over $G^{\Re}$ and the complements are conjugate, being the $\mathfrak{\wp}$-normalizers of $G[\mathbf{1}]$. This theorem has been extended to extensions of abelian $\widetilde{S}_{1}$-groups by hypercentral and hypercyclic groups in [3] and [10], where it is shown that if $G$ is a group with hypercentral (hypercyclic) residual $A$ such that $G / A$ is hypercentral (hypercyclic) and $A$ is an abelian $\widetilde{S}_{1}$-group, then $G$ splits over $A$ and the complements are conjugate.

The result given above for finite groups can be extended to: if $G$ is a finite soluble group whose $\mathfrak{\Im}$-residual $G^{\mathfrak{r}}$ is an $A$-group, then the $\mathfrak{乛}$-normalizers of $G$ complement $G^{\Re}$. (In general, there may be other complements which are not conjugate.) This result has been considered in locally finite groups ([2] and [9]) and we can now give a splitting theorem for groups in which the residual is an $\mathfrak{\subseteq}_{1}-A$-group.

THEOREM 7. Let the group $G$ have hypercentral (hypercyclic) residual $N$ such that $G / N$ is hypercentral (hypercyclic) and $N$ is an $\widetilde{S}_{1}-A$-group. Then $G$ splits over $N$.

Proof. We use induction on the derived length $d$ of $N$. If $d=1$ then $N$ is abelian and the result follows from Theorems $\mathrm{A}$ of [3] and [10]. So we may assume that $d \geqslant 2$. Let $A=N^{(d-1)}$ and $H=N^{(d-2)}$ so that $A=H^{\prime}$ and $A \cap \zeta(H)=1 . A$ is an abelian $\subseteq_{1}$-group and is the hypercentral residual of $H$. By Theorem $\mathrm{A}$ of [3], $H$ splits over $A$ and the complements are conjugate. If $K$ is a complement, then, by the Frattini argument, $A N_{G}(K)=G$. Also $A \cap N_{G}(K)=C_{A}(K)=A \cap \zeta(H)=1$ so that $N_{G}(K) \cong G / A$. The hypercentral (hypercyclic) residual of $G / A$ is $N / A$ and the above isomorphism shows that the hypercentral (hypercyclic) residual of $N_{G}(K)$ is $N \cap N_{G}(K)$. By Corollary 6, N/A and hence $N \cap N_{G}(K)$ is an $\mathfrak{S}_{1}$-group of derived length $d-1$ and so the induction hypothesis shows that $N \cap N_{G}(K)$ has a complement $X$ in $N_{G}(K)$. Then $N X=N N_{G}(K)=G$ and $X \cap N=X \cap N_{G}(K) \cap N=1$ so that $X$ is a complement to $N$ in $G$.

It follows immediately from Theorem 7 that an $\Xi_{1}-A$-group $G$ splits over its derived group. In view of Theorem 2 and the known results for locally finite groups we can make this a little more precise.

THeOrem 8 . Let $G$ be an $\Im_{1}-A$-group. Then $G$ has a characteristic conjugacy class of subgroups characterized by the property of covering all central chief factors and avoiding all eccentric chief factors of $G$. These subgroups complement $G^{\prime}$.

Proof. $G / Z$ is a $\mathfrak{U}$-group and so has a basis normalizer $D / Z$. It is easily checked that $D$ covers every central chief factor of $G$ and avoids every eccentric chief factor.

Conversely, if $E$ is a subgroup with the cover-avoidance property then $E \geqslant Z$ and $E / Z$ is a basis normalizer of $G / Z$ [2, Theorem 2.17].

Clearly $D G^{\prime}=G$ and $D \cap G^{\prime} Z \leqslant Z$; hence $D \cap G^{\prime} \leqslant Z \cap G^{\prime}=1$.

For finite soluble groups, Seitz and Wright [6] proved the complementation theorem under the weaker condition that Sylow $p$-subgroups of the $\mathfrak{\Im}$-residual are quaternion-free if $p=2$ and modular if $p$ is odd. This result depends on the fact that in such groups 
$G^{\text {I }} \cap Z=1$, corresponding to our Theorem 4. It is not clear whether some wider generalization of the splitting theorems can be given by considering groups in which the nilpotent sections satisfy some modularity condition. One of the difficulties will be in considering nilpotent residuals which will not usually have a nilpotent factor group.

\section{REFERENCES}

1. R. W. Carter and T. O. Hawkes, The $\mathfrak{乛}$-normalizers of a finite soluble group, J. Algebra 5 (1967), 175-202.

2. C. J. Graddon, Some generalizations, to certain locally finite groups, of theorems due to Chambers and Rose, Illinois J. Math. 17 (1973), 666-679.

3. B. Hartley and M. J. Tomkinson, Splitting over nilpotent and hypercentral residuals, Math. Proc. Cambridge Philos. Soc. 78 (1975), 215-226. 1972).

4. D. J. S. Robinson, Finiteness conditions and generalized soluble groups, Part 1 (Springer,

5. D. J. S. Robinson, Finiteness conditions and generalized soluble groups, Part 2 (Springer 1972).

6. G. M. Seitz and C. R. B. Wright, On complements of $\mathfrak{乛}$-residuals in finite soluble groups Arch. Math. (Basel) 21 (1970), 139-150.

7. A. L. Smel'kin, Polycyclic groups, Sibirsk. Mat. Ž. 9 (1968), 234-235.

8. D. Taunt, On A-groups, Proc. Cambridge Philos. Soc. 45 (1949), 24-42.

9. M. J. Tomkinson, Formation theory and groups of automorphisms of $\mathfrak{U}$-groups, Proc. Roy Soc. Edinburgh A 76 (1977), 255-265.

10. M. J. Tomkinson, Splitting theorems in abelian-by-hypercyclic groups, J. Austral. Math Soc. Ser A 25 (1978), 71-91.

\section{UNIVERSITY OF GLASGOW}

Department of Mathematics

UNIVERSITY GARDENS

GlasGow

SCOTLAND

G12 8QW 